\title{
universidâd
}

\section{La innovación en la Universidad Nacional Autónoma de Nicaragua, Managua}

\section{Innovation at the National Autonomous University of Nicaragua, Managua}

DOI: http://dx.doi.org/10.5377/uyc.v10i16.6140

César Pereira Morales cesar21pereira2011@yahoo.com Universidad Nacional Autónoma de Nicaragua, Managua.

(C) UNAN-Managua

Recibido: octubre 2017. Aprobado: octubre 2017.

\section{RESUMEN}

La Universidad Nacional Autónoma de Nicaragua, Managua (UNAN-Managua) desde su Rectorado ha venido desarrollando, gestionando e impulsando diferentes iniciativas en pro de mejorar las competencias creativas en los estudiantes de grado de esta Alma Mater. Así lo establecen la Política de investigación e innovación de la UNAN Managua. Es por eso que se han desarrollados esfuerzo en pro de fortalecer una cultura innovadora, entre estos podemos destacar.

Palabras clave: innovación, universidad, iniciativas, competencias.

\begin{abstract}
SUMMARY
The National Autonomous University of Nicaragua, Managua (UNAN-Managua) from its Rectorate has been developing, managing and promoting different initiatives in order to improve the creative competences in the undergraduate students of this Alma Mater. This is established by the Research and Innovation Policy of the UNAN Managua. That is why efforts have been developed to strengthen an innovative culture, among these we can highlight.

Keywords: innovation, university, initiatives, competences.
\end{abstract}

La Universidad Nacional Autónoma de Nicaragua, Managua (UNAN-Managua) desde su Rectorado ha venido desarrollando, gestionando e impulsando diferentes iniciativas en pro de mejorar las competencias creativas en los estudiantes de grado de esta Alma Mater. Así lo establecen la Política de investigación e innovación de la UNAN Managua. Es por eso que se han desarrollados esfuerzo en pro de fortalecer una cultura innovadora, entre estos podemos destacar:

\section{El XIII Taller de Iniciativa de Innovación Global Aplicada en cooperación con la Universidad Grand Valley}

El Taller de Innovación 2017 permitió que los más de 200 participantes idearan alternativas que respondan a problemáticas de las comunidades nicaragüenses; los 31 grupos organizados presentaron 
inicialmente diez ideas lo cual generó más de 300 propuestas. Fue una semana intensa de trabajo en la que estudiantes y docentes visitaron comunidades y barrios estilianos, aplicaron encuestas, realizaron investigaciones, diseñaron prototipos y presentaron sus proyectos ante un jurado evaluador.

Esta actividad se viene realizando desde hace 13 años en la ciudad de Estelí con apoyo de la Iniciativa Global de Innovación Aplicada de la Universidad de Grand Valley, Michigan, Estados Unidos. De ella han surgido importantes proyectos que han obtenido reconocimiento nacional e internacional. Durante el acto de clausura se realizó la presentación de todos los proyectos, la premiación de las mejores propuestas y la evaluación del taller.

Las palabras de clausura estuvieron a cargo del Vicerrector de Investigación, MSc. Javier Pichardo Ramírez, quien indicó que decenas de estudiantes y docentes han pasado por este evento y varios de esos estudiantes ahora son docentes. "El impacto que este evento tiene en la Universidad es extraordinario y debemos transmitirlo al resto de la población estudiantil y docente de la UNAN-Managua”, señaló.

Cada equipo presentó su proyecto ante el jurado que estuvo conformado por delegados de instituciones y organismos nacionales, entre ellas: Jessye Saavedra, del Consejo Nicaragüense de Ciencia y Tecnología (CONICYT); Margarita Cerda y Stephanie Tórrez, de la Cámara de Industria (CADIN); Karla Quiñonez, de la Universidad Nacional de Ingeniería (UNI); Octavio Loáisiga, de la Cámara de Comercio; Julio Baltondano, de ECO Imagen, entre otros.

Las propuestas incluían estudio de mercado, plan financiero, detalles de producción, estructura de marketing; el primer lugar fue para AGROMAP, una aplicación para dispositivos Android que contendrá información sobre el tipo de suelo, su disponibilidad y aprovechamiento agrícola en todo el país. Este proyecto fue elaborado por los jóvenes Karla Vanessa Hernández, de la Facultad de Educación e Idiomas; Douglas Ezequiel Santos Téllez de Ciencias e Ingeniería; Francisco Javier Lainez González, del Programa UNICAM atendido por la FAREM Estelí; Renzon Millers López Navarrete, de Ciencias Económicas; Bryan Lenin Manzony, de Humanidades y Ciencias Jurídicas.

Además, recibieron reconocimiento por haber destacado en el ejercicio del Taxi Pitch, la maestra Ligia Pineda de la FAREM Matagalpa, profesora María Erminia Flores, de Educación e Idiomas y Tania López Toruño, Estudiante de la Facultad de Ciencias Médicas.

Por su parte, el Dr. Paul Lane, principal impulsor de este proyecto, felicitó al equipo de trabajo, el que calificó como “increíble”, puede seguir adelante y generar cambios en Nicaragua. Agradeció el apoyo Universidad y Ciencia, julio-diciembre 2017. Vol. 10, núm. 16, ISSN digital 2313-2760, ISSN impreso 2074-8655 


\section{universidâd}

de los jóvenes de Michigan, de la Dra. Waltraud Beckmann, quien trabajó arduamente en la organización del evento. "La innovación era un sueño del Maestro Cisneros y lo sigue siendo para la Rectora Ramona Rodríguez, por eso se debe seguir adelante sin importar los problemas o las personas que vayan en contra y organizar equipos que puedan ayudarlos a seguir adelante”, expresó el Dr. Paul Lane.

\section{Fondos Concursables para Proyecto de Innovación en el Grado}

El día miércoles 02 de agosto del 2017 en la Dirección de Investigación de Grado de la UNAN Managua, se recibieron 56 propuestas de proyectos de innovación para participar en la Cuarta Convocatoria de Proyectos de Innovación de la UNAN - Managua, mediante el mecanismo de Fondos Concursables 2017, el cual se detalla a continuación: FAREM Estelí con 10 proyectos, Facultad de Ciencias Médicas 10 proyectos, Instituto Politécnico de la Salud 9 proyectos, Facultad de Ciencias Económicas 4 proyectos, FAREM Carazo 1 proyecto, Facultad de Ciencias e Ingeniería 14 proyectos, FAREM Chontales y FAREM Matagalpa con 7 proyectos respectivamente, Facultad de Humanidades y Ciencias Jurídicas 1 proyecto.

Estas 56 propuestas participaron en el proceso de Selección y Ejecución de Proyectos de Innovación de la UNAN - Managua, mediante el mecanismo de Fondos Concursables 2017.

En la semana del 24 al 31 de julio de las 2017 dichas propuestas fueron revisadas en la primera fase de preselección de la Cuarta Convocatoria de los Fondos Concursables para Proyectos de Innovación de la UNAN - Managua y que esta, se limita a una revisión documental, basadas en 13 parámetros establecidos en la normativa de fondos concursables, el cual se llega a la siguiente decisión: $\mathbf{4 8}$ proyectos pasan a la segunda fase correspondiente a la evaluación por panel de expertos externos

Considerándose un mínimo de 80 puntos para ser aprobada cada propuesta se obtuvieron los siguientes resultados:

$\checkmark$ Veinticinco propuestas de proyectos de innovación obtuvieron una calificación entre 80 a 96 puntos

$\checkmark \quad$ Veintitrés propuestas de proyectos de innovación obtuvieron una calificación entre 79 a 45 puntos

Se obtuvieron los siguientes resultados: 25 Proyectos de Innovación adjudicados bajo el fondo concursable, el cual se detalla a continuación: 
La innovación en la UNAN- Managua... César Pereira Morales, pp. 53- 57

\begin{tabular}{|c|c|c|}
\hline $\mathbf{N}^{\mathbf{0}}$ & Título del Proyecto & Facultad a la que pertenece \\
\hline 1 & $\begin{array}{l}\text { Software de asistencia para mantenimiento predictivo } \\
\text { industrial }\end{array}$ & $\begin{array}{l}\text { Facultad de Ciencias e } \\
\text { Ingeniería }\end{array}$ \\
\hline 2 & $\begin{array}{l}\text { Dispositivo termográfico para la detención temprana de } \\
\text { Cáncer }\end{array}$ & IPS \\
\hline 3 & Prótesis Biónica & $\begin{array}{l}\text { Facultad de Ciencias e } \\
\text { Ingeniería }\end{array}$ \\
\hline 4 & NUTRIBARRA & FAREM-Carazo \\
\hline 5 & $\begin{array}{c}\text { Sistema de Información Gerencial, orientado a la publicidad } \\
\text { y promoción de servicios }\end{array}$ & FAREM-Chontales \\
\hline 6 & Espirómetro incentivo Health \&Fitness & Facultad de Ciencias Medicas \\
\hline 7 & Innovación Tecnológica, Organizacional e Institucional & IPS \\
\hline 8 & HEALTH BAND & IPS \\
\hline 9 & Proyecto Mateare Fresh & $\begin{array}{l}\text { Facultad de Ciencias } \\
\text { Económicas }\end{array}$ \\
\hline 10 & PlatHarina & FAREM-Estelí \\
\hline 11 & Proyecto Cosméticos MURAT & $\begin{array}{l}\text { Facultad de Ciencias } \\
\text { Económicas }\end{array}$ \\
\hline 12 & $\begin{array}{l}\text { Elaboración de crema a partir de la capsaicina del chile } \\
\text { cabro }\end{array}$ & $\begin{array}{l}\text { Facultad de Ciencias e } \\
\text { Ingeniería }\end{array}$ \\
\hline 13 & Reactor químico para la elaboración de biodiesel & FAREM-Estelí \\
\hline 14 & Proyecto Natural & $\begin{array}{l}\text { Facultad de Ciencias } \\
\text { Económicas }\end{array}$ \\
\hline 15 & Método Frenkel aplicando a barraras paralelas (MEFABP) & IPS \\
\hline 16 & Proyecto "CIGAR CASE" & IPS \\
\hline 17 & INNOPEDAL & FAREM-Estelí \\
\hline 18 & Entrenamiento dinámico y saludable & Facultad de Ciencias Medicas \\
\hline 19 & $\begin{array}{l}\text { Soldador de punto fabricado con transformador de } \\
\text { microondas }\end{array}$ & FAREM-Estelí \\
\hline 20 & Maquina fundidora de aluminio & FAREM-Estelí \\
\hline 21 & Vinos Alessandro Rouge "Wine Salvatore" & FAREM-Matagalpa \\
\hline 22 & ArtRilax & $\begin{array}{l}\text { Facultad de Ciencias e } \\
\text { Ingeniería }\end{array}$ \\
\hline 23 & Batería de Calor & FAREM-Estelí \\
\hline 24 & AGROMAP & $\begin{array}{l}\text { Facultad de Ciencias } \\
\text { Económicas }\end{array}$ \\
\hline 25 & Procesamiento de Plátano en tostones pre cosidos & FAREM-Chontales \\
\hline
\end{tabular}




\section{universidâd}

De esta manera la UNAN-Managua está trabajando en el fomento de una cultura innovadora y emprendedora, facilitando conocimientos, recursos económicos, redes y vinculaciones con los diferentes sectores del país. La perspectiva que se tiene para el segundo semestre del 2017 en materia de innovación y emprendimiento estará la articulación de los tres grandes procesos, como es la investigación generada en las JUDC 2017, las innovaciones convertidas en prototipos a través de los Fondos Concursables 2017 y la generación de capacidades para la maduración de las ideas de negocios que se puedan luego convertir en una empresa, producto o servicio. 